\title{
RNA Therapy: Current Status and Future Potential
}

\author{
Young-Kook Kim* \\ Department of Biochemistry, Chonnam National University Medical School, Hwasun, Korea
}

\begin{abstract}
Recent studies identified diverse RNAs including noncoding RNAs and their various action mechanisms in the cells. These RNAs regulate a variety of cellular pathways and are therefore expected to be important targets for the treatment of human diseases. Along with their extensive functional studies, RNA-based therapeutic techniques have developed considerably in recent years. After years of research and various trial and error, antisense RNAs and small interfering RNAs-based drugs have been developed and are now being used in the clinic. In addition, active research is ongoing to develop drugs based on RNA aptamer and messenger RNA. Along with the development of these RNA-based drugs, diverse strategies have been developed to transport RNA drugs into the cells efficiently. RNA therapy has many advantages over existing small molecule or monoclonal antibody-based therapies, including its potential to target all genes in the cells. This review will introduce the history of RNA therapy, and explain the basic concepts of RNA therapy and RNA-based drugs on the market or clinical trials. In addition, the future potential of RNA therapy will be discussed.
\end{abstract}

Key Words: RNA, Antisense; RNA, Small Interfering; RNA Interference; Aptamers, Nucleotide; Nanoparticles

This is an Open Access article distributed under the terms of the Creative Commons Attribution Non-Commercial License (http://creativecommons.org/licenses/by-nc/4.0) which permits unrestricted non-commercial use,

distribution, and reproduction in any medium, provided the original work is properly cited.

\section{Article History:}

Received February 5, 2020

Revised February 26, 2020

Accepted February 28, 2020

\section{Corresponding Author:}

Young-Kook Kim

Department of Biochemistry,

Chonnam National University Medical

School, 264 Seoyang-ro,

Hwasun-eup, Hwasun 58128, Korea

Tel: +82-61-379-2777

E-mail: ykk@jnu.ac.kr

\section{INTRODUCTION}

When messenger RNA was discovered about sixty years ago, it was recognized just as an intermediate to transmit the genetic information between DNA and the ribosomes. After several key discoveries including RNA with enzymatic function (ribozyme) and the regulatory RNAs including microRNA and long noncoding RNAs, RNA is now accepted as the key molecules which are involved in nearly all biological pathways. While researchers were discovering the diverse roles of RNA, many scientists also noticed the substantial advantages of using RNA as therapeutic molecules. Their efforts led to the development of diverse RNA-based drugs over the last twenty years. With the ongoing extensive research into RNA therapy, it is expected that we will obtain diverse strategies for treating human diseases. This review will summarize the timeline of the important discoveries and the key topics of RNA therapy. Diverse RNA-based drugs and their delivery method will also be discussed.

\section{TIMELINE OF KEY DISCOVERIES IN RNA THERAPY}

There have been many essential discoveries that led to the establishment of the RNA therapy field (Fig. 1). After the discovery of DNA as genetic material, the key question was how the genetic information is translated into protein. The 'unstable messengers' which carry information from the DNA in the nucleus into the protein-synthesizing machinery (ribosomes) in the cytoplasm were discovered by Sydney Brenner and his colleagues. ${ }^{1,2}$ This messenger RNA (mRNA), along with the transfer RNA (tRNA) were identified at a similar time ${ }^{3}$ and the ribosomal RNA (rRNA) which comprises the ribosome, had been recognized as one of the three main RNA classes in the cells.

Because the RNA is a nucleic acid that can bind sequence-specifically to the other nucleic acids, a chemically synthesized antisense RNA can be employed to suppress the expression of target RNA. The initial experiment was performed more than forty years ago to block the replication of the Rous sarcoma virus and therefore suppress the cellular transformation. ${ }^{4}$ This approach is very straight- 


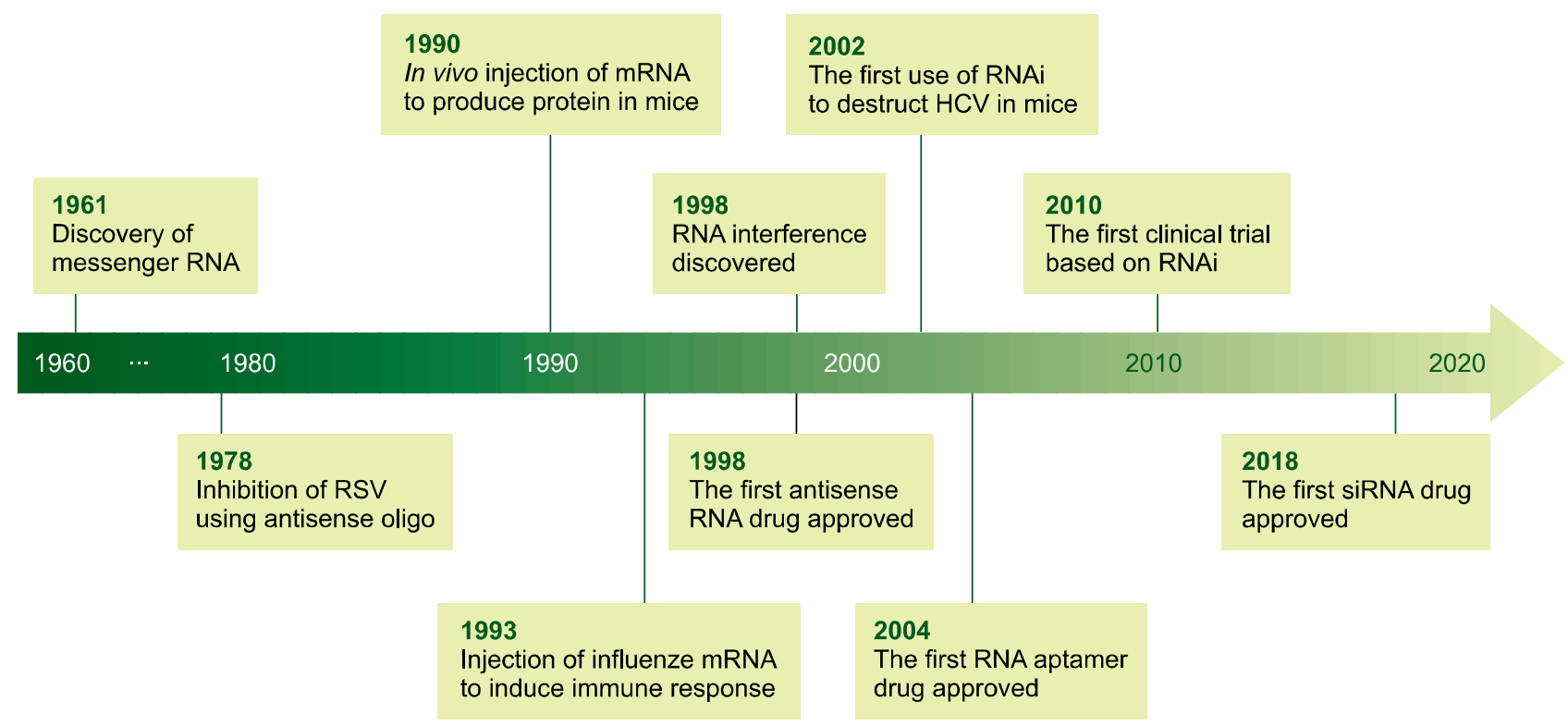

FiG. 1. Timeline of key discoveries in RNA therapy. See the text for details.

forward in that the required information to make the antisense oligonucleotides is only the target sequence. Although diverse chemical modifications are applied to antisense oligonucleotides to make RNA-based drugs nowadays, the basic principle, which is the production of complementary sequences for the binding into target nucleic acids, is identical.

More than ten years after the first utilization of antisense oligonucleotides, the potential of mRNA for the treatment was reported. The researchers showed that direct injection of mRNA into mouse skeletal muscle without any delivery material resulted in the production of the corresponding protein. ${ }^{5}$ Soon after this study, other groups synthesized the mRNA code for the influenza nucleoprotein, encapsulated it into the liposome, and injected these complexes into mice. ${ }^{6}$ This treatment resulted in the induction of cytotoxic T lymphocyte suggesting this approach is valid to develop an RNA-based vaccine.

The discovery of RNA interference (RNAi) opened a new era in the application of this methodology into both the academic and therapeutic fields. ${ }^{7}$ Whereas the researchers in the academic field obtained an unprecedented tool to discover the function of an unknown gene by exploiting the inherently existing cellular mechanism, the medical industry acquired an easy way to eliminate disease-causing types of RNA. Whereas the antisense oligonucleotide techniques rely on the single-strand RNA, which binds complementarily to their targets, the molecule utilized in the RNAi pathway is small interfering RNA (siRNA), a shortsized double-stranded RNA. This makes the RNAi tools require a relatively tougher method to penetrate the cells but with fewer molecules (discussed in the next section).

In the same year when the RNAi was discovered, the first drug based on antisense oligonucleotide, fomivirsen, was approved by the Food and Drug Administration (FDA) in the United States (Table 1). ${ }^{8}$ This antisense oligonucleotide drug works through binding to the complementary sequence of mRNAs produced from the cytomegalovirus (CMV) genome. It was developed to treat CMV retinitis, a disease with inflammation of the retina, in immunocompromised patients. However, it was withdrawn from the market because CMV retinitis cases dropped significantly due to the development of antiretroviral therapy.

The second RNA-based drug called pegaptanib was approved by the FDA in 2004 (Table 1). Pegaptanib, the first RNA aptamer drug, was developed to treat the wet type of age-related macular degeneration. ${ }^{9}$ It works by specific binding to vascular endothelial growth factor (VEGF), an essential factor for the formation and permeability of blood vessels. ${ }^{10}$ Through the blocking of the VEGF action, pegaptanib reduces the growth of the blood vessels within the eye.

After the discovery of RNAi, it was widely adopted for research in the laboratory, especially for cell-based studies. In the meantime, the therapeutic potential of RNAi was rapidly proved from initial studies regarding applying the siRNA to disrupt the sequence of the hepatitis $C$ virus (HCV) in adult mice. ${ }^{11}$ The potent effect of siRNA was shown through the transgene expression of a part of the HCV genome in this study.

The first clinical trial based on siRNA was reported in $2010 .^{12}$ In this trial, siRNA encapsulated in the nanoparticle was administrated systemically to the patients with skin cancer melanoma. By confirming the existence of a mRNA cleavage fragment in the tumor biopsies from patients, which is evidently produced from the siRNAmediated RNAi mechanism, ${ }^{13}$ this study demonstrated that siRNA administration can result in the inhibition of a specific target gene in human.

Although the potential of RNAi as research tools was evi- 
TABLE 1. List of clinically approved RNA drugs by the United States Food and Drug Administration

\begin{tabular}{|c|c|c|c|c|c|c|}
\hline Category & Drug & $\begin{array}{l}\text { Brand } \\
\text { name }\end{array}$ & $\begin{array}{l}\text { Approved } \\
\text { year }\end{array}$ & $\begin{array}{l}\text { Target } \\
\text { molecule }\end{array}$ & $\begin{array}{l}\text { Treatment } \\
\text { result }\end{array}$ & $\begin{array}{l}\text { Target } \\
\text { disease }\end{array}$ \\
\hline \multicolumn{7}{|c|}{ Antisense RNA (single-stranded RNA) } \\
\hline & Fomivirsen & Vitravene & 1998 & IE2 mRNA & $\begin{array}{l}\text { Binds to IE2 mRNA and blocks its trans- } \\
\text { lation }\end{array}$ & CMV retinitis \\
\hline & Mipomersen & Kynamro & 2013 & ApoB mRNA & $\begin{array}{l}\text { Binds to ApoB mRNA and induces its } \\
\text { degradation by RNase } \mathrm{H}\end{array}$ & $\begin{array}{l}\text { Familial } \\
\text { hypercholesterolemia }\end{array}$ \\
\hline & Nusinersen & Spinraza & 2016 & SMN2 mRNA & $\begin{array}{l}\text { Modulates the alternative splicing of } \\
\text { SMN2 mRNA and increases the SMN } \\
\text { protein level }\end{array}$ & $\begin{array}{l}\text { Spinal muscular } \\
\text { atrophy }\end{array}$ \\
\hline & Eteplirsen & Exondys 51 & 2016 & $\begin{array}{l}\text { Dystrophin } \\
\text { mRNA }\end{array}$ & $\begin{array}{l}\text { Induces the exclusion of exon } 51 \text { of dy- } \\
\text { strophin mRNA during splicing to pro- } \\
\text { duce a functional protein }\end{array}$ & $\begin{array}{l}\text { Duchenne muscular } \\
\text { dystrophy }\end{array}$ \\
\hline & Inotersen & Tegsedi & 2018 & $\begin{array}{l}\text { Transthyretin } \\
\text { mRNA }\end{array}$ & $\begin{array}{l}\text { Binds to Transthyretin mRNA and in- } \\
\text { duces its degradation by RNase } \mathrm{H}\end{array}$ & $\begin{array}{l}\text { Hereditary } \\
\text { transthyretin } \\
\text { amyloidosis }\end{array}$ \\
\hline & Golodirsen & Vyondys 53 & 2019 & $\begin{array}{l}\text { Dystrophin } \\
\text { mRNA }\end{array}$ & $\begin{array}{l}\text { Induces the exclusion of exon } 53 \text { of dy- } \\
\text { strophin mRNA during splicing to pro- } \\
\text { duce a functional protein }\end{array}$ & $\begin{array}{l}\text { Duchenne muscular } \\
\text { dystrophy }\end{array}$ \\
\hline \multicolumn{7}{|c|}{ Small interfering RNA (double-stranded RNA) } \\
\hline & Patisiran & Onpattro & 2018 & $\begin{array}{l}\text { Transthyretin } \\
\text { mRNA }\end{array}$ & $\begin{array}{l}\text { Suppresses the hepatic production of } \\
\text { transthyretin protein through RNA in- } \\
\text { terference }\end{array}$ & $\begin{array}{l}\text { Hereditary } \\
\text { transthyretin } \\
\text { amyloidosis }\end{array}$ \\
\hline & Givosiran & Givlaari & 2019 & ALAS1 mRNA & $\begin{array}{l}\text { Reduces the hepatic production of ALAS1 } \\
\text { protein through RNA interference }\end{array}$ & $\begin{array}{l}\text { Acute hepatic } \\
\text { porphyria }\end{array}$ \\
\hline \multicolumn{7}{|c|}{ RNA aptamer } \\
\hline & Pegaptanib & Macugen & 2004 & VEGF protein & $\begin{array}{l}\text { Binds specifically to the } 165 \text { isoform of } \\
\text { VEGF and blocks its function }\end{array}$ & $\begin{array}{l}\text { Age-related macular } \\
\text { degeneration }\end{array}$ \\
\hline
\end{tabular}

dent and promising results from initial clinical trials were obtained, however, it takes quite a long time before a drug based on this technology is approved. Whereas the target RNAs were suppressed dramatically in the cell-based study, researchers realized that it was not so easy to deliver the siRNAs into the desired region of the body. Through experimentation, researchers noticed that the siRNA-based molecules injected into the body accumulated in the liver. ${ }^{14}$ Consequently, many companies tried to develop drugs that could target the diseases caused by a problem in this organ.

Finally, the first siRNA drug, patisiran, was approved by FDA in 2018 (Table 1). This drug exerts its effect by targeting the 3 ' untranslated region of transthyretin mRNA. ${ }^{15}$ The transthyretin protein primarily made in the liver is clustered as amyloid deposits in the body of patients with hereditary transthyretin-mediated amyloidosis. ${ }^{16}$ By reducing the amount of transthyretin protein, patisiran can alleviate the symptoms of this disease. The second siRNA drug, givosiran, was approved in the last year. ${ }^{17}$ Considering the accumulated knowledge from recent research and many numbers of ongoing clinical trials, the development of diverse RNA-based drugs is expected in the near future.

\section{CATEGORIES OF RNA THERAPY}

\section{Antisense RNAs (single-stranded RNAs)}

Based on the identity of RNA drugs, RNA therapy can be categorized into three broad areas (Fig. 2). The first category includes the RNA molecules that target nucleic acids, either DNA or RNA. These RNA molecules are further divided into two subcategories, single-stranded antisense RNAs and double-stranded RNAs (Fig. 2A and 2B). The antisense RNAs have complementary sequences to the target molecules, such as mRNAs. By binding to the target, they can modulate the splicing of pre-mRNAs, induce the degradation of target mRNAs mainly through the RNase Hmediated degradation, or block the translation of mRNAs into proteins.

One of the RNA drugs approved by the FDA in 2013, mipomersen, works by inducing the $\mathrm{RNase} \mathrm{H}$-mediated degradation of apolipoprotein $\mathrm{B}(\mathrm{ApoB}) \mathrm{mRNAs} .{ }^{18}$ Since ApoB is a critical protein in lipid metabolism, this drug can be used to treat familial hypercholesterolemia. ${ }^{19}$ Another RNA drug based on antisense mechanism is inotersen. This drug, approved by FDA in 2018, is used to treat hereditary transthyretin amyloidosis. By binding to transthyretin mRNA, inotersen reduces its translation into protein..$^{20}$ The first RNA-based drug, fomivirsen, also works by a similar mechanism, where it binds to immediate-early 2 (IE2) mRNA produced from the CMV genome and blocks its translation. $^{21}$

Two other antisense RNA drugs approved in 2016, nusinersen and eteplirsen, modulate the splicing of premRNAs. They were intended to treat the inherited neuro- 
A Antisense RNA (single-stranded RNA)
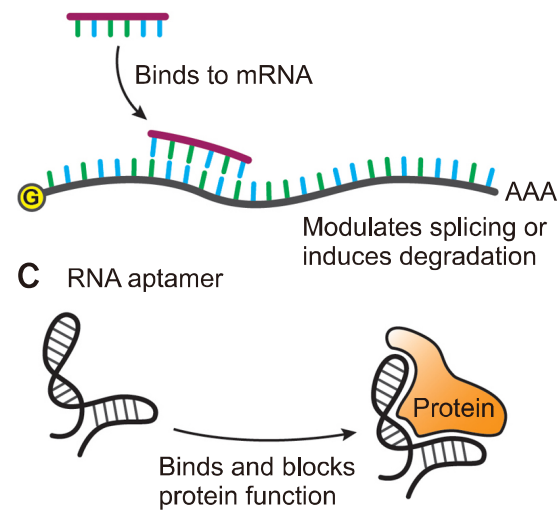

B Small interfering RNA (double-stranded RNA)

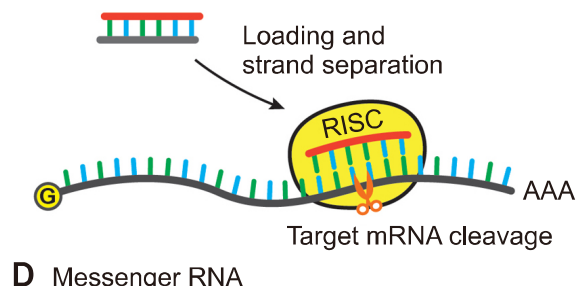

Messenger RNA

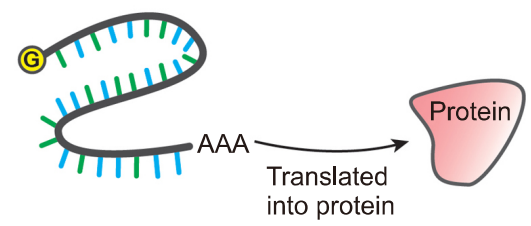

FiG. 2. Types of RNA therapy. (A) Antisense RNA (single-stranded RNA). The single-stranded antisense RNA is designed to bind to pre- or mature mRNA. After binding, it modulates the splicing of pre-mRNA or induces the degradation of mRNA. It can also inhibit the translation of mRNA into protein. (B) Small interfering RNA (double-stranded RNA). The small interfering RNA (siRNA) is introduced as a double-stranded form. After loading into the RNA-induced silencing complex (RISC), one strand is removed after strand separation. The siRNA-RISC complex binds to target mRNA sequence-specifically and cleaves the mRNA (depicted as scissor) inducing its degradation. (C) RNA aptamer. The RNA aptamer can bind to a specific protein and block its function. (D) Messenger RNA. After the messenger RNA (mRNA) is introduced into the cells, cellular machinery including the ribosome translates its information into a protein, the final product that can work as an enzyme or antigen.

muscular diseases of children, spinal muscular atrophy and Duchenne muscular dystrophy, respectively. Nusinersen modulates the splicing of survival of motor neuron 2 (SMN2) pre-mRNA. ${ }^{22}$ In a normal splicing reaction, the exon 7 of SMN2 pre-mRNA is excluded, and the protein product from this mRNA has low stability. However, nusinersen binds to the SMN2 pre-mRNA and induces the inclusion of exon 7 , and consequently, this increases the stability of the translated protein. In contrast, eteplirsen induces the exclusion of exon 51 of dystrophin pre-mRNA. ${ }^{23}$ In patients with Duchenne muscular dystrophy, the reading frame of dystrophin mRNA is broken due to the mutation at this exon and no functional dystrophin protein is made. By binding to the dystrophin pre-mRNA, eteplirsen induces the alternative splicing reaction which excludes exon 51, and shorter but functional dystrophin proteins can be produced. The recently approved antisense oligonucleotides drug to treat the same disease, golodirsen, also induces the skipping of exon 53 of dystrophin pre-mRNA. ${ }^{24}$

\section{Small interfering RNAs (double-stranded RNAs)}

The double-stranded siRNA drugs exert their function through the cellular pathway, RNAi (Fig. 2B). ${ }^{25}$ After being loaded into the RNA-induced silencing complex (RISC), the key complex of the RNAi pathway, the double strands of siRNA are separated and one strand is discarded. The remaining RNA strand in the RISC binds to the target mRNA sequence-specifically, and the argonaute- 2 protein in the RISC cleaves the target mRNA inducing its degradation. Until now, only two siRNA drugs have been approved by the FDA although many drugs are in clinical trials. The patisiran, described in the previous section, is a chemically modified siRNA drug to treat hereditary transthyretin- mediated amyloidosis. It induces the cleavage of transthyretin mRNA by binding to the 3 ' untranslated region of this mRNA. ${ }^{15}$

Givosiran is the second siRNA drug approved by the FDA in 2019. This drug is intended to treat acute hepatic porphyria, a rare inherited genetic disease. It binds to and suppresses the translation of delta aminolevulinic acid synthase 1 (ALAS1) mRNA, thereby reducing the neurotoxic intermediates in this disease. ${ }^{17}$

Another siRNA-based drug inclisiran, which is expected to be on the market soon, shows the great durability of siRNA in the body, which is one of the key advantages of this type of drug. Inclisiran was developed to treat patients with familial hypercholesterolemia. It binds to and cleaves the mRNA sequence of proprotein convertase subtilisin/ kexin type 9 (PCSK9), which is a target to lower the level of low-density lipoprotein (LDL) cholesterol. ${ }^{26}$ The data from the clinical trial showed that the effect of inclisiran lasts more than six months with only a single-dose treatment. ${ }^{27}$ This extremely long efficiency is possible because after being loaded into the RISC, the siRNA is protected from nucleases and can operate multiple times, although only $1-2 \%$ of siRNAs entered into the cells through endosome can escape into the cytosol, and then are loaded into RISC. ${ }^{28,29}$ This result confirms the great potential of siRNAbased drugs for therapeutic uses.

\section{RNA aptamers}

The second category of RNA therapy is the RNA drug which targets proteins. This drug includes the RNA aptamer, which binds and modulates the function of proteins (Fig. 2C). As described above, pegaptanib is an RNA aptamer, which binds to VEGF protein. ${ }^{9}$ It was developed to 
treat the wet type of age-related macular degeneration. The RNA aptamer has a high affinity to the target protein similar to antibodies. Compared to antibodies, however, RNA aptamer is small in size and has additional advantages including improved transport into the cells and lower cost. Dozens of RNA aptamers are in the clinical trial stages. $^{30}$

\section{Messenger RNAs}

The third category of RNA therapy is RNA drugs which are translated into proteins. Thus, mRNA with chemical modifications to increase its stability are used as drugs. After the introduction into the cells, the mRNA is translated into protein and exerts its function (Fig. 2D). In present, this drug is intended to be used as a personalized cancer vaccine or a vaccine for infectious diseases. ${ }^{31}$ For example, the samples of a patient with cancer are obtained from blood or tissue. After comparing the genomic sequence of the cancer samples to that of normal tissue, the cancer-specific mutations of the patient are identified. Among those mutations, the possible sequences which can be used for antigen production are selected and the mRNAs are synthesized based on this prediction. These mRNAs are finally injected into the tumor region, and the antigen-specific immune cells can be expanded to eliminate the tumor cells. The great potential of this procedure is that personalized immunotherapy is possible within a relatively short time. In the case of small molecule-based drugs, personalized therapy is very difficult.

\section{DELIVERY IN RNA THERAPY}

One great advantage of RNA therapy is that RNA drug can be used to target 'undruggable' molecules which are otherwise hard to target using small molecule-based drugs. From an estimation, only one-fifth of proteins can be targeted by commonly used drugs including small molecules and antibodies. ${ }^{32}$ Moreover, it is impossible to target noncoding RNAs, which are not translated into proteins, using traditional small molecules or monoclonal antibodies. Since RNA drugs can bind specifically to all types of RNAs, the cellular molecules which have RNA forms in their intermediate stages during production (protein) or in their final form (noncoding RNAs) can be targeted.

Compared to small molecules, however, general RNA drugs are much bigger in size and have a high electric charge. This makes the intracellular delivery of RNA molecules in their native forms across cell membranes quite difficult. The delivery of RNA molecules into the right tissues is also another big huddle. In addition, RNAs are easily degraded due to the ubiquitous RNases inside the body. Thus, RNA, in its naked form, is difficult to use in therapy (Fig. 3).

To solve the problem of intracellular delivery, researchers have utilized several delivery strategies. The nanoparticle is one of the strategies to introduce RNA drugs into the cell efficiently (Fig. 3). Nanoparticles for RNA delivery

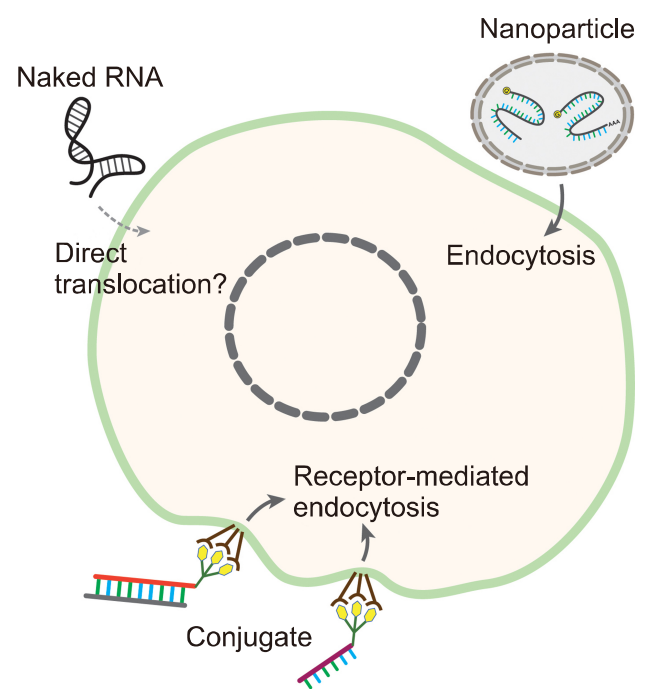

FiG. 3. The delivery method in RNA therapy. The RNA drugs can be introduced into the cells as a naked form, although the efficiency is very low due to the high electric charge and the large size of RNAs. This problem can be solved by encapsulating the RNAs into lipid or other types of the nanoparticle. In another way, a chemical conjugate is attached to the RNA molecule, and this conjugate is recognized by a specific cell surface receptor. These nanoparticles and RNA-conjugate complexes are introduced through endocytosis and exert their effects.

can be synthesized in a variety of forms. ${ }^{33}$ In one strategy, RNAs can be encapsulated inside the lipid bilayers with a positive surface charge. This lipid nanoparticle protects the RNAs and increases their half-life. Moreover, lipid bilayers increase the efficiency of endocytosis into the cells and help them escape the endosomes. Patisiran is one of the RNA drugs encapsulated inside the lipid nanoparticle. Many other RNA-based drugs now on the clinical trials also use lipid nanoparticles for their delivery. ${ }^{34}$ An additional strategy based on nanoparticle includes the method of mixing negatively charged RNAs with positively charged polymers. ${ }^{35}$ Since the liver is the main filtering tissue that traps nanoparticles, a large proportion of nanoparticle-based RNA drugs target the diseases which can be cured by delivering those drugs into the liver. ${ }^{14}$

Another major strategy to deliver RNA drugs is their conjugates. Since the conjugate binds to the specific receptor on the surface of the cells, it can be used to deliver the covalently bound RNA molecule into a specific cell type. Once the conjugate is bound to the cell surface receptor, the RNA-conjugate complex is introduced into the cells through receptor-mediated endocytosis (Fig. 3). One of the conjugates clinically used in RNA therapy is $\mathrm{N}$-acetylgalactosamine (GalNAc). This conjugate is recognized by the hepatocyte-specific asialoglycoprotein receptor. ${ }^{36}$ Givosiran is the first-ever FDA-approved RNA drug based on GalNAc conjugate technology. The development of other types of conjugates is underway. A recent report showed that by conjugating the ligand of the glucagon-like peptide- 1 receptor, the antisense oligonucleotide was delivered to the 
pancreatic $\beta$-cells effectively. ${ }^{37}$ Since less excipient materials are required than using nanoparticle technology, the conjugate-based delivery shows a lower risk of toxicity.

\section{PERSPECTIVE FOR THE FUTURE OF RNA THERAPY}

The development of diverse RNA-based drugs reported recently shows the great potential of this field. Based on the knowledge accumulated so far, it is expected that new RNA-based drugs will emerge to treat various diseases that have no reported treatment method. As described above, one of the great advantages of RNA-based therapy is that it is possible to target both protein-coding and noncoding RNAs. For the protein-coding RNAs, RNA-based drugs can be used to modulate previously undruggable targets. Another advantage of RNA therapy is that researchers can design the drugs rapidly. After the phosphate backbone and ribose structures of RNA-targeting antisense oligonucleotides or siRNAs are established, the remaining step is just changing the sequence of RNAs so as to make the complementary binding of these RNA drugs to target molecules. The accumulated knowledge from the hard work that have been performed to control the characteristic of RNAs will make the design of RNA drugs even faster and easier.

Another important advantage of RNA therapy is its long-lasting effects when using siRNAs for RNA drugs. As shown from the recent clinical trial, the expression of target gene PCSK9 was suppressed efficiently, and consequently, the decreased level of LDL cholesterol was maintained even after six months from the treatment. ${ }^{27}$ This long-lasting effect of siRNA-based drugs is beneficial for patients whom it may not feasible to receive frequent treatments. Since small molecule-based drugs, which are still primarily used nowadays, do not last very long, more research into the development of siRNA-based drugs will improve the weakness of this type of drug.

The delivery of RNA-based drugs was one of the main challenges in this field. However, this problem has been considerably circumvented thanks to the recent development of the diverse delivery platforms. The results from recently approved drugs and those in the clinical trials showed that RNA drugs can be targeted to the liver with high efficiency. However, we still do not have a good strategy to deliver RNA drugs to the tissue other than the liver efficiently. Therefore, one of the essential studies in the future is to develop a way to deliver the drugs into these other tissues to treat diseases which have less relation to the liver.

We are witnessing remarkable progress in RNA therapy with great advances in RNA research. With the well-established field of small molecule or antibody-based drugs, we now have a new class of RNA-based drugs. By developing these diverse kinds of therapeutics together, it can be expected that diverse diseases with no treatment method today will be conquered in the near future.

\section{ACKNOWLEDGEMENTS}

This study was supported by grants from the Basic Science Research Program through the National Research Foundation of Korea (NRF), funded by the Ministry of Science, ICT \& Future Planning (NRF-2018R1A2B6001104).

\section{CONFLICT OF INTEREST STATEMENT}

None declared.

\section{REFERENCES}

1. Brenner S, Jacob F, Meselson M. An unstable intermediate carrying information from genes to ribosomes for protein synthesis. Nature 1961;190:576-81.

2. Gros F, Hiatt H, Gilbert W, Kurland CG, Risebrough RW, Watson JD. Unstable ribonucleic acid revealed by pulse labelling of Escherichia coli. Nature 1961;190:581-5.

3. Weinberg RA, Penman S. Small molecular weight monodisperse nuclear RNA. J Mol Biol 1968;38:289-304.

4. Zamecnik PC, Stephenson ML. Inhibition of Rous sarcoma virus replication and cell transformation by a specific oligodeoxynucleotide. Proc Natl Acad Sci U S A 1978;75:280-4.

5. Wolff JA, Malone RW, Williams P, Chong W, Acsadi G, Jani A, et al. Direct gene transfer into mouse muscle in vivo. Science 1990; 247(4949 Pt 1):1465-8.

6. Martinon F, Krishnan S, Lenzen G, Magné R, Gomard E, Guillet $\mathrm{JG}$, et al. Induction of virus-specific cytotoxic T lymphocytes in vivo by liposome-entrapped mRNA. Eur J Immunol 1993;23: 1719-22.

7. Fire A, Xu S, Montgomery MK, Kostas SA, Driver SE, Mello CC. Potent and specific genetic interference by double-stranded RNA in Caenorhabditis elegans. Nature;391:806-11.

8. Roehr B. Fomivirsen approved for CMV retinitis. J Int Assoc Physicians AIDS Care 1998;4:14-6.

9. Gragoudas ES, Adamis AP, Cunningham ET Jr, Feinsod M, Guyer DR; VEGF Inhibition Study in Ocular Neovascularization Clinical Trial Group. Pegaptanib for neovascular age-related macular degeneration. N Engl J Med 2004;351:2805-16.

10. Chang JH, Garg NK, Lunde E, Han KY, Jain S, Azar DT. Corneal neovascularization: an anti-VEGF therapy review. Surv Ophthalmol 2012;57:415-29.

11. McCaffrey AP, Meuse L, Pham TT, Conklin DS, Hannon GJ, Kay MA. RNA interference in adult mice. Nature 2002;418:38-9.

12. Davis ME, Zuckerman JE, Choi CH, Seligson D, Tolcher A, Alabi CA, et al. Evidence of RNAi in humans from systemically administered siRNA via targeted nanoparticles. Nature 2010;464: 1067-70.

13. Carthew RW, Sontheimer EJ. Origins and mechanisms of miRNAs and siRNAs. Cell 2009;136:642-55.

14. Lorenzer C, Dirin M, Winkler AM, Baumann V, Winkler J. Going beyond the liver: progress and challenges of targeted delivery of siRNA therapeutics. J Control Release 2015;203:1-15.

15. Adams D, Gonzalez-Duarte A, O'Riordan WD, Yang CC, Ueda M, Kristen AV, et al. Patisiran, an RNAi therapeutic, for hereditary 
transthyretin amyloidosis. N Engl J Med 2018;379:11-21.

16. Adams D, Koike H, Slama M, Coelho T. Hereditary transthyretin amyloidosis: a model of medical progress for a fatal disease. Nat Rev Neurol 2019;15:387-404.

17. Sardh E, Harper P, Balwani M, Stein P, Rees D, Bissell DM, et al. Phase 1 trial of an RNA interference therapy for acute intermittent porphyria. N Engl J Med 2019;380:549-58.

18. Wong E, Goldberg T. Mipomersen (kynamro): a novel antisense oligonucleotide inhibitor for the management of homozygous familial hypercholesterolemia. P T 2014;39:119-22.

19. Marais AD. Familial hypercholesterolaemia. Clin Biochem Rev 2004;25:49-68.

20. Benson MD, Waddington-Cruz M, Berk JL, Polydefkis M, Dyck PJ, Wang AK, et al. Inotersen treatment for patients with hereditary transthyretin amyloidosis. N Engl J Med 2018;379:22-31.

21. Geary RS, Henry SP, Grillone LR. Fomivirsen: clinical pharmacology and potential drug interactions. Clin Pharmacokinet 2002;41:255-60.

22. Wurster CD, Ludolph AC. Nusinersen for spinal muscular atrophy. Ther Adv Neurol Disord 2018;11:1756285618754459.

23. Lim KR, Maruyama R, Yokota T. Eteplirsen in the treatment of Duchenne muscular dystrophy. Drug Des Devel Ther 2017;11: 533-45.

24. Rodrigues M, Yokota T. An Overview of recent advances and clinical applications of exon skipping and splice modulation for muscular dystrophy and various genetic diseases. Methods Mol Biol 2018;1828:31-55.

25. Wilson RC, Doudna JA. Molecular mechanisms of RNA interference. Annu Rev Biophys 2013;42:217-39.

26. Hooper AJ, Burnett JR. Anti-PCSK9 therapies for the treatment of hypercholesterolemia. Expert Opin Biol Ther 2013;13:429-35.

27. Fitzgerald K, White S, Borodovsky A, Bettencourt BR, Strahs A,
Clausen V, et al. A highly durable RNAi therapeutic inhibitor of PCSK9. N Engl J Med 2017;376:41-51.

28. Hutvágner G, Zamore PD. A microRNA in a multiple-turnover RNAi enzyme complex. Science 2002;297:2056-60.

29. Gilleron J, Querbes W, Zeigerer A, Borodovsky A, Marsico G, Schubert U, et al. Image-based analysis of lipid nanoparticlemediated siRNA delivery, intracellular trafficking and endosomal escape. Nat Biotechnol 2013;31:638-46.

30. Kaur H, Bruno JG, Kumar A, Sharma TK. Aptamers in the therapeutics and diagnostics pipelines. Theranostics 2018;8:4016-32.

31. Pardi N, Hogan MJ, Porter FW, Weissman D. mRNA vaccines a new era in vaccinology. Nat Rev Drug Discov 2018;17:261-79.

32. Finan C, Gaulton A, Kruger FA, Lumbers RT, Shah T, Engmann $\mathrm{J}$, et al. The druggable genome and support for target identification and validation in drug development. Sci Transl Med 2017;9:eaag1166.

33. Donahue ND, Acar H, Wilhelm S. Concepts of nanoparticle cellular uptake, intracellular trafficking, and kinetics in nanomedicine. Adv Drug Deliv Rev 2019;143:68-96.

34. Kulkarni JA, Cullis PR, van der Meel R. Lipid nanoparticles enabling gene therapies: from concepts to clinical utility. Nucleic Acid Ther 2018;28:146-57.

35. Kaczmarek JC, Kowalski PS, Anderson DG. Advances in the delivery of RNA therapeutics: from concept to clinical reality. Genome Med 2017;9:60.

36. Huang X, Leroux JC, Castagner B. Well-defined multivalent ligands for hepatocytes targeting via asialoglycoprotein receptor. Bioconjug Chem 2017;28:283-95.

37. Ämmälä C, Drury WJ 3rd, Knerr L, Ahlstedt I, Stillemark-Billton $\mathrm{P}$, Wennberg-Huldt C, et al. Targeted delivery of antisense oligonucleotides to pancreatic $\beta$-cells. Sci Adv 2018;4:eaat3386. 